\title{
BMJ Open Cross-sectional study of coeliac autoimmunity in a population of Vietnamese children
}

Sara Zanella, ${ }^{1}$ Luigina De Leo, ${ }^{2}$ Le Nguyen-Ngoc-Quynh, ${ }^{3}$ Bo Nguyen-Duy, ${ }^{3}$ Tarcisio Not, ${ }^{2}$ Mai Tran-Thi-Chi, ${ }^{3}$ Son Phung-Duc, ${ }^{3}$ Hai Le-Thanh, ${ }^{3}$ Cristina Malaventura, ${ }^{1}$ Serena Vatta, ${ }^{2}$ Fabiana Ziberna, ${ }^{2}$ Martina Mazzocco, ${ }^{1}$ Stefano Volpato, ${ }^{1}$ Lan Phung-Tuyet, ${ }^{3}$ Huong Le-Thi-Minh, ${ }^{3}$ Caterina Borgna-Pignatti ${ }^{1}$

To cite: Zanella S, De Leo L, Nguyen-Ngoc-Quynh L, et al. Cross-sectional study of coeliac autoimmunity in a population

of Vietnamese children. BMJ Open 2016;6:e011173. doi:10.1136/bmjopen-2016011173

- Prepublication history for this paper is available online. To view these files please visit the journal online (http://dx.doi.org/10.1136/ bmjopen-2016-011173).

SZ and LDL contributed equally.

Received 20 January 2016 Revised 18 March 2016 Accepted 22 March 2016

\section{CrossMark}

${ }^{1}$ Department of Medical Sciences/Pediatrics, University of Ferrara, Ferrara, Italy

${ }^{2}$ Institute for Maternal and Child Health—IRCCS "Burlo Garofolo", Trieste, Italy

${ }^{3}$ National Hospital of Pediatrics, Hanoi, Vietnam

Correspondence to Professor Caterina BorgnaPignatti; c.borgna@unife.it

\section{ABSTRACT}

Objective: The prevalence of coeliac disease (CD) in Vietnam is unknown. To fill this void, we assessed the prevalence of serological markers of $C D$ autoimmunity in a population of children in Hanoi.

Setting: The outpatient blood drawing laboratory of the largest paediatric hospital in North Vietnam was used for the study, which was part of an international project of collaboration between Italy and Vietnam.

Participants: Children having blood drawn for any reason were included. Exclusion criteria were age younger than 2 years, acquired or congenital immune deficiency and inadequate sample. A total of 1961 children (96\%) were enrolled (838 females, 1123 males, median age 5.3 years).

Outcomes: Primary outcome was the prevalence of positive autoimmunity to both IgA antitransglutaminase antibodies (anti-tTG) assessed with an ELISA test and antiendomysial antibodies (EMA). Secondary outcome was the prevalence of $C D$ predisposing human leucocyte antigens (HLA) (HLA DQ2/8) in the positive children and in a random group of samples negative for IgA anti-tTG.

Results: The IgA anti-tTG test was positive in 21/1961 (1\%; $95 \% \mathrm{Cl} 0.61 \%$ to $1.53 \%$ ); however, EMA antibodies were negative in all. HLA DQ2/8 was present in $7 / 21(33 \% ; 95 \% \mathrm{Cl} 14.5 \%$ to $56.9 \%)$ of the anti-tTG-positive children and in $72 / 275(26 \%$; $95 \% \mathrm{Cl}$ $21 \%$ to $32 \%$ ) of those who were negative.

Conclusions: Coeliac autoimmunity is rare in Vietnam, although prevalence of HLA DQ2/8 is similar to that of other countries. We hypothesise that the scarce exposure to gluten could be responsible for these findings.

\section{INTRODUCTION}

Coeliac disease (CD) is a chronic small-intestinal immune-mediated enteropathy precipitated by exposure to dietary gluten in genetically predisposed individuals bearing the second class human leucocyte antigen (HLA) DQ2/DQ8 haplotypes. ${ }^{1} \mathrm{CD}$ is

\section{Strengths and limitations of this study}

- This is the first research on coeliac disease autoimmunity in Vietnam.

- HLA typing for DQ2 and DQ8 of the antitransglutaminase antibodies (anti-tTG)-positive children and of a proportion of those who were negative was carried out.

- A detailed questionnaire was used to collect quantitative information about gluten consumption to the best possible accuracy.

- The target population was composed of children presenting to a hospital laboratory and therefore by definition not completely healthy.

- A small-intestinal biopsy could not possibly be performed and therefore we do not know if histological lesions were present in the few patients at risk.

characterised by the presence of a variable combination of clinical manifestations including intestinal and extraintestinal symptoms such as diarrhoea, abdominal pain, failure to thrive and anaemia. CD-specific antibodies comprise antitransglutaminase antibodies (anti-tTG), antiendomysial antibodies (EMA) and antibodies against deamidated forms of gliadin peptides. ${ }^{2}$ The sensitivity of anti-tTG and EMA is about $93 \%$, whereas specificity has been reported to be 97\% for anti-tTG and $99 \%$ for EMA.

The prevalence of CD reported in Europe and the USA averages $1 \%$ in children and adults. ${ }^{3-5}$ A few studies have been performed on symptomatic individuals in China and India ${ }^{6}$ but, with the exception of a screening in Malaysian adults, ${ }^{7}$ the prevalence of $\mathrm{CD}$ in the Asia-Pacific region and, more specifically in Vietnam, is still unknown. The World Gastroenterology Organization and the Asian Pacific Association of Gastroenterology recommend establishing the prevalence of $\mathrm{CD}$ across that region in order to increase 
awareness among physicians and patients. ${ }^{8}$ When untreated, the disease can cause permanent growth failure and poor bone development, and, according to some studies, it can facilitate the development of autoimmune disorders such as diabetes and thyroiditis, infertility and even cancer. ${ }^{9}{ }^{10}$ A twofold to threefold excess in all-cause mortality among patients with untreated CD, compared with the general population, has been reported. ${ }^{11} 12$

While wheat is the staple cereal of most Caucasian populations, the diet of many populations in Asia and South-East Asia is based on rice. ${ }^{9} 13$ Wheat-based products, however, are becoming more common with urbanisation and rising incomes in areas of Asia that were once considered traditional rice-eating regions. Changes in infant feeding patterns in Asian countries might increase the prevalence of CD. We hypothesised that eating mainly rice would protect one from developing coeliac autoimmunity.

In order to establish the prevalence of $\mathrm{CD}$ in the Vietnamese paediatric population, we tested a large number of children, using the serum anti-tTG as a screening test. The children who tested positive for anti-tTG were evaluated with the EMA test and for the CD-related HLA. A randomly selected group of children who had tested negative for anti-tTG and EMA were typed for CD-related HLA.

\section{MATERIALS AND METHODS}

\section{Study design}

The study was designed by the University of Ferrara, Italy, in collaboration with the National Hospital of Paediatrics (NHP) in Hanoi, Vietnam, which are partners in an international project of the University of Ferrara. In addition, the Institute for Clinical Research of the University of Trieste, Italy, participated in the research project and performed all the laboratory tests. The sample size was calculated on an estimation of $0.75 \%$ prevalence of CD. ${ }^{14}$ Considering a $99 \% \mathrm{CI}$ and a precision of $0.5 \%$, the estimated sample size was $1976 .{ }^{15}$ We added $5 \%$ to the sample size to compensate for any attrition.

The NHP is the second largest paediatric hospital in Southeast Asia and the large daily influx of children allowed us to enrol the necessary number in a short period of time. The children, aged 2-18 years, who presented to the laboratory of the NHP to have blood drawn for any reason between February 2 and 14, 2015, were included in the study. Exclusion criteria were: age younger than 2 years, a diagnosis of malignancy and chemotherapy or treatment with immunosuppressants, including corticosteroids. In fact, testing for anti-tTG and EMA in children below 2 years of age results in poor sensitivity, ${ }^{2}$ and immunosuppression could also decrease the sensitivity of the tests.

Blood sampling was carried out at the NHP: two tubes were obtained from each participant, one for serum and one for whole blood. We searched for IgA anti-tTG in all the children included in the study. All the positive children were then tested for EMA and HLA DQ2/DQ8. Total IgA concentration was measured in samples with IgA anti-tTG absorbance ranging from 0 to 0.140 , as previously reported, ${ }^{16}$ and serum samples with IgA deficiency (IgA serum concentration $<7 \mathrm{mg} / \mathrm{dL}$ ) were tested for IgG anti-tTG.

Furthermore, a random selection of children who tested negative for anti-tTG and EMA were also evaluated for CD-related HLA. According to the ESPGHAN criteria, ${ }^{2}$ a participant is defined as being at risk for having $\mathrm{CD}$ when positive for anti-tTG and EMA in the presence of HLA DQ2/DQ8.

A questionnaire was used to collect demographic data (sex, date of birth, current therapies), information on signs and symptoms known to be related to CD (recent symptoms of abdominal discomfort or fatigue), and on the approximate amount of gluten consumed weekly (noodles, bread or snacks: never, once a day, more or less than once a week).

\section{Serological assays and HLA typing}

Serum samples were examined in duplicate at the NHP laboratory for IgA anti-tTG, using an ELISA assay (Eu-tTG, Eurospital, Trieste, Italy) according to the manufacturer's instructions (normal values $<9 \mathrm{U} / \mathrm{mL}$ ). Quantitative determination of human IgA in serum was carried out in Italy using an immunoassay (Roche/Hitachi Cobas c system, Indianapolis, Indiana, USA), following the manufacturer's instructions. Serum EMA were evaluated by indirect immunofluorescence on cryostat sections of human umbilical cord, as previously described. ${ }^{3}$

The susceptibility alleles for $\mathrm{CD}$ were determined by PCR with allele-specific primers identifying HLA DQ2 and DQ8, using an Eu-Gene-Risk kit (Eurospital, Trieste, Italy). The kit serves to identify all DQ2-positive participants carrying both DQ2.5 (HLA-DQA1*05, DQB1*02 in cis with DR3 or in trans with the DR5/DR7 haplotypes) and DQ2.2 heterodimers (HLA-DQA $1 * 02$, DQB $1 * 02$, DRB $1 * 07)$, and those positive for DQ8 (HLA-DQA1*03, DQB1*03:02, DRB1*04).

\section{Statistical analysis}

Continuous data were presented as mean \pm SD for normally distributed parameters, and median and IQR for skewed variables. Dichotomous variables were presented as frequency and percentage.

\section{Ethical considerations}

Written informed consent was obtained from the children's parents before proceeding with the tests.

\section{RESULTS}

\section{Study population}

The parents of 2045 children agreed to participate in the study. Eighty-four children $(4 \%)$ were excluded due to inadequate serum samples (63 children) or to 
exclusion criteria (15 with leukaemia, 3 in chemotherapy, 1 in radiotherapy, 2 under 2 years of age). Nineteen hundred and sixty-one children $(96 \%)$ were enrolled in the study (838 females, 1123 males, median age 5.3 years and IQR 4-7.5 years). Reasons for having blood drawn included an array of general paediatric diseases: respiratory tract infections, fever, gastroenteritis, cough, hepatitis, thalassaemia major, anaemia, abdominal pain, nephrotic syndrome, glomerulonephritis, stunted growth, thyroiditis, diabetes, arthritis, asthma, tuberculosis, urinary tract infection, dengue, Henoch-Schonlein purpura and immune thrombocytopenia.

Twenty per cent of the children $(387 / 1961)$ presented with gastrointestinal symptoms (266 of recurrent abdominal pain, 87 anorexia, 58 diarrhoea). One hundred and twenty-eight others were being worked up because of failure to thrive. Exposure to gluten was reported by $88 \%$ of the patients' parents. Four per cent of them ate foods containing gluten every day, $40 \%$ at least once a week and $56 \%$ less than once a week.

\section{Anti-tTG, EMA and HLA typing}

Twenty-one children out of 1961 (8 females, 13 males) tested positive for IgA anti-tTG $(1 \%$; $95 \%$ CI $0.61 \%$ to $1.53 \%)$ and 17 of the 21 had a history of eating gluten. However, EMA antibodies were negative in all of them. Seven of the $21(33 \%)$ carried the CD-related HLA (table 1$)$, but only in 2 ( $0.1 \%$ of the total population) was the titre of anti-tTG antibodies higher than three times the upper limit of normal (positive predictive value $95 \%) .{ }^{17}$ One patient had an IgA anti-tTG titre 10 times higher than the upper limit of normal values but his HLA DQ2/8 was negative. HLA DQ2/8 was present in $7 / 21 \quad(33 \% ; 95 \%$ CI $14.5 \%$ to $56.9 \%)$ of the anti-tTG-positive children. The HLA DQ2/8 was measured also in 275/1961 (14\%) children (selected by means of a computational random number generator) who had tested negative for anti-tTG and EMA, and $72 / 275$ (26\%; $95 \%$ CI $21 \%$ to $32 \%$ ) demonstrated presence of the HLA DQ2/8.

IgA anti-tTG absorbance ranging from 0 to 0.140 was present in 162/1961 children (8\%) and 5/162 (3\%: 3 females, 2 males, median age 6.4 years) had total IgA deficiency. These five children were tested and were found to be negative for IgG anti-tTG. The results of the study are shown in figure 1 .

\section{DISCUSSION}

This is the first study on the prevalence of coeliacspecific antibodies among Vietnamese children.

$\mathrm{CD}$ is considered to be rare in the Asia-Pacific region. Therefore, we screened a large sample of children who had not previously been diagnosed with $\mathrm{CD}$, by means of sensitive and specific sequential serological tests. Overall, 21 children were found to be anti-tTG IgA positive, but the EMA test was negative in all, and only $7 / 21$ were positive for CD-related HLA. Only two children were potentially affected by $\mathrm{CD}$, having high levels of anti-tTG and being DQ2/DQ8 positive. However, both

\begin{tabular}{|c|c|c|c|c|c|c|c|c|}
\hline Patient & Sex & $\begin{array}{l}\text { Age } \\
\text { (years) }\end{array}$ & $\begin{array}{l}\text { IgA anti-tTG } \\
(\mathrm{U} / \mathrm{mL})\end{array}$ & $\begin{array}{l}\text { IgA anti-tTG } \\
\text { absorbance }\end{array}$ & HLA & $\begin{array}{l}\text { EMA } \\
(+/-)\end{array}$ & $\begin{array}{l}\text { Gluten } \\
\text { exposure }\end{array}$ & $\begin{array}{l}\text { Gluten consumption } \\
\text { frequency }\end{array}$ \\
\hline V731 & $M$ & 4 & 9 & 0.628 & $\mathrm{~N}$ & - & Yes & $>1$ per week \\
\hline V348 & $F$ & 10 & 9 & 0.648 & $\mathrm{~N}$ & - & Yes & $<1$ per week \\
\hline V1755 & $M$ & 11 & 10 & 0.659 & $\mathrm{~N}$ & - & Yes & $>1$ per week \\
\hline V1057 & $M$ & 8 & 10 & 0.632 & DQ8 & - & No & \\
\hline V703 & $M$ & 6 & 10.5 & 0.659 & $\mathrm{~N}$ & - & Yes & $>1$ per week \\
\hline V525 & $F$ & 17 & 12 & 0.712 & $\mathrm{~N}$ & - & No & \\
\hline V715 & $\mathrm{F}$ & 6 & 13.5 & 0.731 & $\mathrm{~N}$ & - & Yes & $<1$ per week \\
\hline V800 & $M$ & 6 & 14 & 0.779 & DQ2.5 & - & Yes & $<1$ per week \\
\hline V949 & $M$ & 4 & 14 & 0.733 & DQ2.5/8 & - & Yes & $<1$ per week \\
\hline V431 & $F$ & 4 & 16 & 0.794 & DQ2.5 & - & Yes & $>1$ per week \\
\hline V736 & $M$ & 3 & 16 & 0.803 & $\mathrm{~N}$ & - & No & \\
\hline V1417 & $F$ & 9 & 17 & 0.846 & $\mathrm{~N}$ & - & Yes & $<1$ per week \\
\hline V1872 & $F$ & 5 & 18.5 & 0.832 & $\mathrm{~N}$ & - & Yes & $<1$ per week \\
\hline V1521 & $M$ & 6 & 21.5 & 1.021 & DQ2.5 & - & Yes & $>1$ per week \\
\hline V517 & $M$ & 9 & 37 & 1.3 & $\mathrm{~N}$ & - & Yes & $<1$ per week \\
\hline V510 & $F$ & 9 & 40 & 1.303 & $\mathrm{~N}$ & - & Yes & $<1$ per week \\
\hline V170 & $M$ & 8 & 45 & 1.341 & DQ2.5 & - & Yes & $>1$ per week \\
\hline V1965 & $M$ & 7 & 54 & 1.692 & $\mathrm{~N}$ & - & Yes & $>1$ per week \\
\hline V1037 & $F$ & 8 & 64 & 1.852 & $\mathrm{~N}$ & - & Yes & $>1$ per week \\
\hline V148 & $M$ & 9 & 74 & 2.195 & DQ2.2 & - & Yes & $<1$ per week \\
\hline V963* & $M$ & 10 & 105 & 3.045 & $\mathrm{~N}$ & - & No & \\
\hline
\end{tabular}

${ }^{*} \operatorname{IgA}$ anti-tTG titre was 10 times higher than the upper limit of normal values but HLA DQ2/8 was negative.

Anti-tTG, antitransglutaminase antibodies; EMA, antiendomysial antibodies; F, female; HLA N, HLA DQ2/8 negative; M, male. 


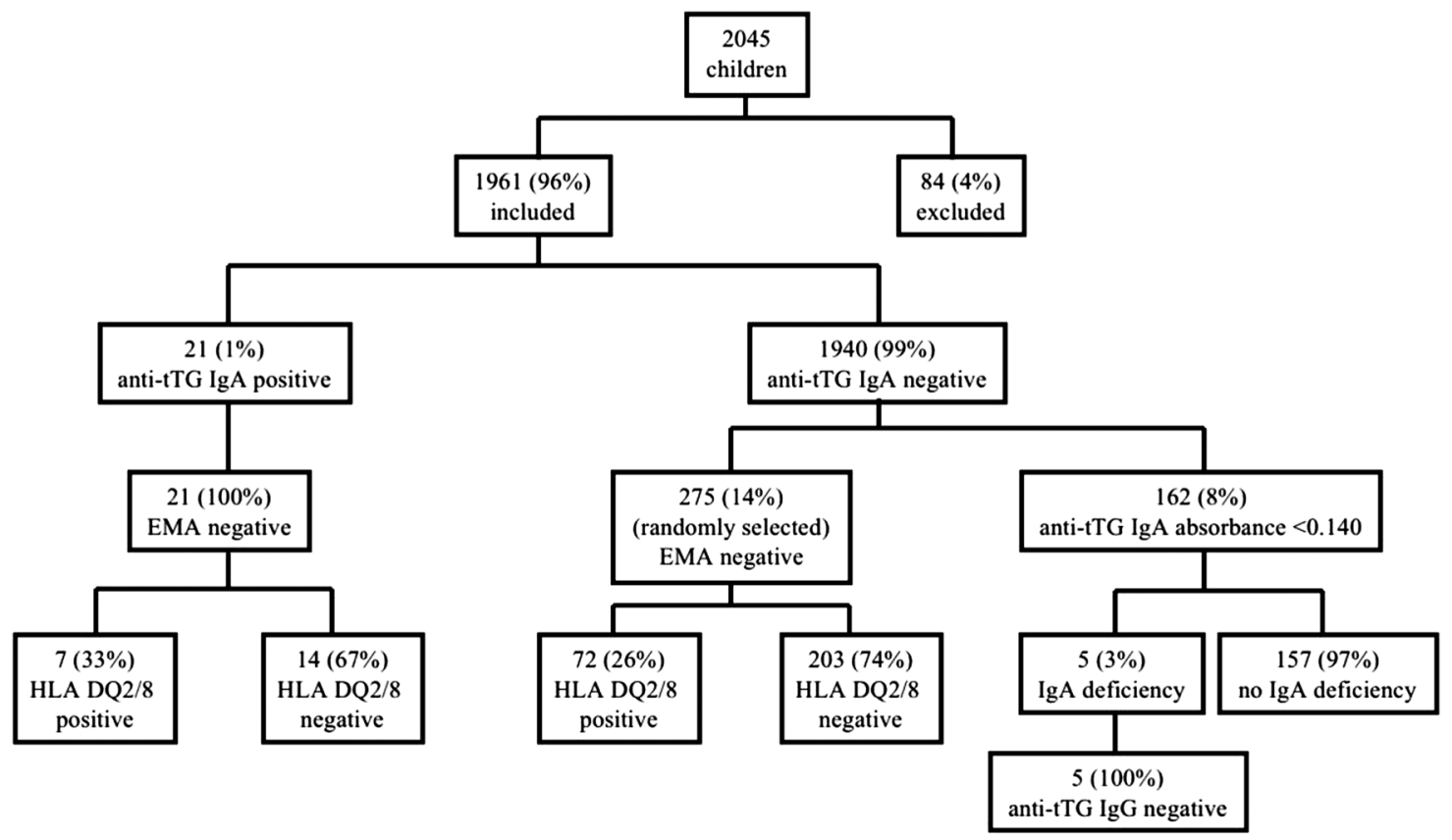

Figure 1 Flow chart showing results of the study. Anti-tTG, antitransglutaminase antibodies; EMA, antiendomysial antibodies.

were EMA negative. According to the literature and the recent ESPGHAN ${ }^{2}$ and BSPGHAN $^{18}$ guidelines for CD diagnosis, the EMA test is considered a gold standard immunological biomarker as sensitive as, but more specific than, anti-tTG, the increase of which might be caused also by parasitosis. ${ }^{19}$ The remainder of the children who tested positive for DQ2 and DQ8 were anti-tTG and EMA negative.

All the screening studies performed so far in the Eastern Hemisphere have identified the presence of CD autoimmunity in variable percentages. In India, CD has been well recognised, especially in the north, and two population-based studies revealed a prevalence of 0.3 to $1.04 \% .{ }^{20}$ According to a meta-analysis, ${ }^{21}$ the number of reported cases of $\mathrm{CD}$ is extremely low in China, although a study of children with chronic diarrhoea showed a histologically proven frequency of CD equalling $12 \% .^{22}$ Preliminary data from Japan and Singapore suggest the existence of $\mathrm{CD}$ also in these countries. ${ }^{6} \mathrm{~A}$ study from Malaysia reported a prevalence of $1.9 \%$ in adult females and $0.4 \%$ in males as demonstrated by positive $\mathrm{IgA} / \mathrm{IgG}$ antigliadin antibodies, $\operatorname{IgA} / \operatorname{IgG}$ anti-tTG and EMA. ${ }^{7}$

The pathogenesis of $\mathrm{CD}$ is linked to involvement of the HLA molecules, DQ2 or DQ8, which present gluten antigens to specific T-cells. Their presence is a condition not sufficient but necessary condition to develop CD. The typical HLA alleles were present in $26 \%$ of the Hanoi children examined, a number similar to the percentages found in most populations. ${ }^{13}$ The low prevalence of $\mathrm{CD}$ autoimmunity in our northern Vietnamese paediatric population is therefore somewhat surprising, especially in consideration of the fact that HLA genotyping suggests that the risk for $\mathrm{CD}$ exists also in Vietnam.

Possible explanations for this finding could be the young median age of the children screened ( 5.3 years) or the scarce and late introduction of gluten. A recent multicentre, prospective European study demonstrated that, although late introduction of gluten did not decrease the risk of developing CD in children with predisposing conditions, it delayed the onset of the disease. ${ }^{23}$ Approximately half of the Vietnamese children that we examined ate gluten-containing foods less than once a week, and $10 \%$ did not eat gluten at all. This fact could be responsible for the rarity of coeliac autoimmunity.

IgA deficiency occurs more frequently in patients with $\mathrm{CD}(1.30 \%)$ than in the general population $(0.13$ to $0.25 \%)$, a fact that might cause false-negative results. ${ }^{24}$ In our study, the children who were considered as being possibly affected by total or partial IgA deficiency ${ }^{16}$ on the basis of low IgA anti-tTG absorbance were tested with IgG anti-tTG and found to be negative.

In our population, there was a prevalence of males. This difference reflects the male-to-female ratio, which averages 120:100 (45\% female) in the Red River Delta (Hanoi City) and 110:100 (47\% female) in the rest of the northern areas. ${ }^{25}$

Our results are similar to those recently reported from Colombia, where healthy individuals and those affected by autoimmune disorders were both tested with anti-tTG and EMA. Among patients with autoimmune disorders, seven individuals tested positive or weakly positive for anti-tTG, but IgA EMA were negative in all cases. ${ }^{26}$ 
Strengths and limitations of the study

The strength of our study lies in the fact that this is the first research on CD autoimmunity in Vietnam; in the large number of children examined by ELISA for anti-tTG; in the further testing by EMA of positive sera, and in the HLA typing for DQ2 and DQ8 of the anti-tTG-positive children. Also, in children affected by total or partial IgA deficiency, anti-tTG IgG were measured. The amount of gluten introduced with food was estimated by means of a detailed questionnaire with the best possible accuracy.

The study was performed on children having blood drawn as outpatients at a hospital laboratory, who therefore were not completely healthy. However, if anything, this potential selection bias should have increased the prevalence of CD in our sample. The median age of children was 5 years, and we do not know if $\mathrm{CD}$ autoimmunity will develop with passing years. A small-intestinal biopsy could not possibly be performed and therefore the presence of histological lesions in the two patients (V170, V148) with high anti-tTG antibody concentration carrying the CD-related HLA cannot be completely excluded. Both had been exposed to dietary gluten.

\section{Future developments}

New studies on the Southeast Asian population might clarify whether the prevalence of $\mathrm{CD}$ increases with age and if there is a strict correlation with the amount of gluten introduced with the diet. It will be of interest to follow children on a completely gluten-free diet and compare them with children from the same area whose diet includes gluten-containing foods.

\section{CONCLUSIONS}

None of the 1961 Vietnamese children examined were positive for coeliac autoimmunity on the basis of positivity for anti-tTG and EMA. The extremely low prevalence of $\mathrm{CD}$ in this large population of children could be due to low exposure to gluten coupled with the young age of the children.

Acknowledgements The authors thank the nurses at the NHP of Hanoi without whose help this study would not have been possible, and the parents of the children, who patiently answered many questions. They also acknowledge the secretarial help of Ms Cinzia Tonioli.

Contributors CBP, TN, HLTM, LPT and CM were involved in study design. SZ, LDL, MM, BND, SPD, LNNQ and MTTC were involved in data collection. CBP, TN, SZ, LDL, CM, StV, MTTC, SeV, FZ and HLT were involved in data analysis. CBP, TN, SZ, LDL, CM, StV and LPT were involved in data interpretation. CBP, TN, SZ, LDL, CM and StV were involved in writing.

Funding Financial support for the study was provided by the Paediatric Unit of the University of Ferrara, Italy, and by the Institute for Maternal and Child Health-Burlo Garofolo, Trieste, Italy. SZ was the recipient of a fellowship of the Atlante programme for international collaboration.

Competing interests None declared.

Patient consent Obtained.
Ethics approval National Hospital of Pediatrics, Hanoi and Comitato Etico Unico della Provincia di Ferrara, and the University Hospital of Ferrara.

Provenance and peer review Not commissioned; externally peer reviewed.

Data sharing statement No additional data are available.

Open Access This is an Open Access article distributed in accordance with the Creative Commons Attribution Non Commercial (CC BY-NC 4.0) license, which permits others to distribute, remix, adapt, build upon this work noncommercially, and license their derivative works on different terms, provided the original work is properly cited and the use is non-commercial. See: http:// creativecommons.org/licenses/by-nc/4.0/

\section{REFERENCES}

1. Ludvigsson JF, Leffler DA, Bai JC, et al. The Oslo definitions for coeliac disease and related terms. Gut 2013;62:43-52.

2. Husby S, Koletzko S, Korponay-Szabó IR, et al. European Society for Pediatric Gastroenterology, Hepatology, and Nutrition guidelines for the diagnosis of coeliac disease. J Pediatr Gastroenterol Nutr 2012;54:136-60.

3. Tommasini A, Not T, Kiren V, et al. Mass screening for coeliac disease using antihuman transglutaminase antibody assay. Arch Dis Child 2004;89:512-15.

4. Hoffenberg EJ, MacKenzie T, Barriga KJ, et al. A prospective study of the incidence of childhood celiac disease. J Pediatr 2003;143:308-14. http://www.ncbi.nlm.nih.gov/pubmed/14517510 (accessed 3 Jun 2015).

5. Mäki M, Mustalahti K, Kokkonen J, et al. Prevalence of celiac disease among children in Finland. N Engl J Med 2003;348:2517-24.

6. Makharia GK. Celiac disease screening in southern and East Asia. Dig Dis 2015;33:167-74.

7. Yap TW, Chan WK, Leow AH, et al. Prevalence of serum celiac antibodies in a multiracial Asian population-a first study in the young Asian adult population of Malaysia. PLOS ONE 2015;10: e0121908.

8. Makharia GK, Mulder CJ, Goh KL, et al., World Gastroenterology Organization-Asia Pacific Association of Gastroenterology Working Party on Celiac Disease. Issues associated with the emergence of coeliac disease in the Asia-Pacific region: a working party report of the World Gastroenterology Organization and the Asian Pacific Association of Gastroenterology. J Gastroenterol Hepatol 2014;29:666-77.

9. Lionetti E, Catassi C. New clues in celiac disease epidemiology, pathogenesis, clinical manifestations, and treatment. Int Rev Immunol 2011;30:219-31.

10. Ventura A, Magazzù G, Greco L. Duration of exposure to gluten and risk for autoimmune disorders in patients with celiac disease. SIGEP Study Group for Autoimmune Disorders in Celiac Disease. Gastroenterology 1999;117:297-303. http://www.ncbi.nlm.nih.gov/ pubmed/10419909 (accessed 2 Nov 2015).

11. Corrao G, Corazza GR, Bagnardi V, et al. Mortality in patients with coeliac disease and their relatives: a cohort study. Lancet 2001;358:356-61.

12. Biagi F, Corazza GR. Mortality in celiac disease. Nat Rev Gastroenterol Hepatol 2010;7:158-62.

13. Cummins AG, Roberts-Thomson IC. Prevalence of celiac disease in the Asia-Pacific region. J Gastroenterol Hepatol 2009;24:1347-51.

14. Fasano A, Berti I, Gerarduzzi T, et al. Prevalence of celiac disease in at-risk and not-at-risk groups in the United States: a large multicenter study. Arch Intern Med 2003;163:286-92.

15. Cicchitelli G. Statistica: principi e metodi. Pearson Paravia Bruno Mondadori, 2008.

16. Fernández E, Blanco C, García S, et al. Use of low concentrations of human $\lg \mathrm{A}$ anti-tissue transglutaminase to rule out selective $\lg \mathrm{A}$ deficiency in patients with suspected celiac disease. Clin Chem 2005;51:1014-16.

17. Hill PG, Holmes GKT. Coeliac disease: a biopsy is not always necessary for diagnosis. Aliment Pharmacol Ther 2008;27:572-7.

18. Murch S, Jenkins $H$, Auth M, et al. Joint BSPGHAN and Coeliac UK guidelines for the diagnosis and management of coeliac disease in children. Arch Dis Child 2013;98:806-11.

19. Giersiepen K, Lelgemann M, Stuhldreher N, et al. Accuracy of diagnostic antibody tests for coeliac disease in children: summary of an evidence report. J Pediatr Gastroenterol Nutr 2012;54:229-41.

20. Makharia GK, Verma AK, Amarchand R, et al. Prevalence of celiac disease in the northern part of India: a community based study. J Gastroenterol Hepatol 2011;26:894-900. 
21. Yuan J, Gao J, Li X, et al. The tip of the 'celiac Iceberg' in China: a systematic review and meta-analysis. PLOS ONE 2013;8: e81151.

22. Wang XQ, Liu L, Xu CD, et al. Celiac disease in children with diarrhoea in 4 cities in China. $J$ Pediatr Gastroenterol Nutr 2011;53:368-70.

23. Lionetti E, Castellaneta S, Francavilla R, et al, SIGENP (Italian Society of Pediatric Gastroenterology, Hepatology, and Nutrition) Working Group on Weaning and CD Risk. Introduction of gluten,
HLA status, and the risk of celiac disease in children. N Engl J Med 2014;371:1295-303.

24. Chow MA, Lebwohl B, Reilly NR, et al. Immunoglobulin A deficiency in celiac disease. J Clin Gastroenterol 2012;46:850-4.

25. http://www.gso.gov.vn/. General Statistics Office Of Vietnam. http:// www.gso.gov.vn/Default_en.aspx?tabid=491 (accessed 1 Jun 2015)

26. Parra-Medina R, Molano-Gonzalez N, Rojas-Villarraga A, et al. Prevalence of celiac disease in Latin America: a systematic review and meta-regression. PLOS ONE 2015;10:e0124040. 